\title{
Qualitätsdaten als Grundlage für Vertragswettbewerb
}

Die Gesundheitsreformen der letzten Jahre führten zu einer marktwirtschaftlichen und wettbewerbsorientierten Neuausrichtung des Gesundheitssektors, indem sie unter anderem auf der Beschaffungsseite eine Lockerung des Kontrahierungszwanges vorsahen. Zielstellung dieses Vertragswettbewerbs ist, mit unterschiedlichen Steuerungselementen die Prozesse der Prävention, Diagnose, Therapie und Rehabilitation fachund/oder sektorenübergreifend zu planen, zu begleiten, zu bewerten und nach medizinisch qualitativen und wirtschaftlichen Gesichtspunkten zu optimieren.

\section{Versorgungsqualität}

$\checkmark$

Verbunden mit einer stärkeren Wettbewerbsorientierung geht auch die Forderung der Kostenträger nach einer vereinbarten Behandlungssicherheit sowie gesteigerten Versorgungsqualität einher. Dies auch vor dem Hintergrund, da durch selektive Vertragsoptionen die Versorgungsqualität in einen unmittelbaren Zusammenhang mit dem Kostenträger gebracht wird.

Eine hohe Versorgungsqualität benötigt zur Sicherung von Behandlungserfolgen die Einbindung von strukturierten Routinen. Basis hierfür muss eine bessere Verzahnung der einzelnen Versorgungsbereiche untereinander sein. Dies setzt fach- und sektorenübergreifende Koopera- tionen der einzelnen Akteure voraus. Ziel ist die ganzheitliche Betrachtung des Krankheits- und Versorgungsereignisses.

Und auch die Versicherten und Patienten fordern dies ein: Eine Versichertenbefragung der Techniker Krankenkasse (TK) hat ergeben, dass eindeutig Qualität sowie Service im Rahmen der ärztlichen Behandlung erwartet werden.

Im Weiteren erhebt der souveräne Patient zunehmend den Anspruch auf Qualitätstransparenz.

\section{Qualitätsziele im Einzelvertragsbereich}

In den ersten Jahren der integrierten Versorgung war vornehmlich die Überführung bzw. Substitution von klar abgrenzbaren Leistungen aus der Regelversorgung in den Bereich der Einzelverträge nach $\S 140$ a ff. SGB V zu beobachten (z.B. stationsersetzendes Operieren, Leistungskomplexe im Bereich Endoprothetik und Kardiochirurgie). Auch wenn der Anteil von Verträgen, in welchen Krankenhäuser als vertragsschließende Partner auftreten, kontinuierlich abnimmt (1. Quartal 2005 79\%, 4. Quartal 2008 48,3\%), haben entsprechende Verträge nach wie vor eine hohe Bedeutung.

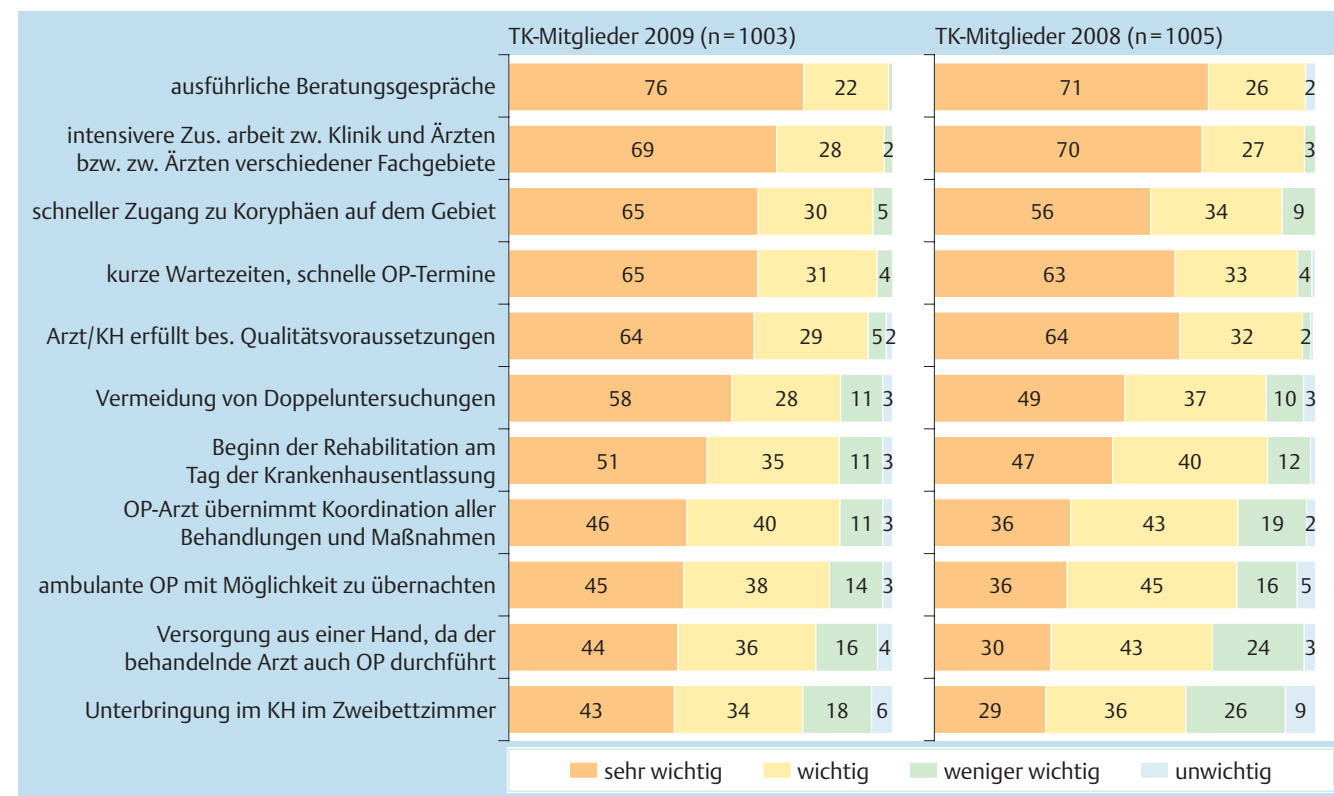

Abb. 1 TK-Trendmonitor 2009, TK-repräsentativ, $n=1003$ Fälle (Quelle: Techniker Krankenkasse 2009).
K. Rupp

Qualitätsmanagement

Schlüsselwörter

Benchmark

Kosteneffektivität

Qualität

- Selektiv-Verträge

Versorgungsmanagement

Keywords

benchmarking

cost-effectiveness

quality

selective contracts

managed care

Institut

Techniker Krankenkasse, Hamburg

Bibliografie

DOI $10.1055 / \mathrm{s}-0029-1242675$ Dtsch Med Wochenschr 2009; 134: S319-S320 - (c) Georg Thieme Verlag KG Stuttgart . New York · ISSN 0012-0472

Korrespondenz Klaus Rupp Techniker Krankenkasse Bramfelder Straße 140 22305 Hamburg Tel. 040/6909-1932 eMail klaus.rupp@tk-online.de 
So stellt die integrierte Versorgung derzeit für Krankenhausträger und Krankenkassen die einzige Option der „freien“ Leistungsvereinbarung dar, um durch entsprechende Verträge mit dem „prefered Provider" eine Versorgungssteuerung nach Qualitätsaspekten anzustreben und durch eine Vereinbarung zusätzlicher Leistungsmengen Wirtschaftlichkeitsvorteile zu generieren.

\section{Praxisbeispiel der TK: Ergebnis- orientierte Vergütung in der Herzchirurgie}

Im Bereich der Herzchirurgie bietet die TK bereits seit einigen Jahren qualitativ hochwertige Versorgungsangebote mit ausgewählten Krankenhäusern sowie Rehabilitationseinrichtungen und niedergelassenen Kardiologen an. Diese Vertragskonzepte entwickelt sie laufend weiter. Nachdem die TK in der Herzchirurgie mit Kliniken Komplexpauschalen vereinbart hat, gibt es nun in diesem Bereich erste Verträge mit einer erfolgsorientierten Vergütung:

Das Krankenhaus meldet bestimmte Qualitätsparameter an die Bundesgeschäftsstelle für Qualitätssicherung (BQS). Die beteiligen Vertragskrankenhäuser haben sich der TK gegenüber verpflichtet, ihre BQS-Werte offenzulegen. Sind die Vertragskrankenhäuser erfolgreicher als der Bundesdurchschnitt, erhalten sie zusätzlich einen Bonus auf die vereinbarte Vergütung.

Auch der Patient profitiert von solchen Verträgen, indem die Kliniken ihre Behandlungsverfahren immer wieder neu unter Beweis stellen müssen.

Vor dem Hintergrund der Patientenorientierung fließen zunehmend auch $\mathrm{Er}$ kenntnisse aus Patientenbefragung in die Wahl des Vertragspartners und Ausgestaltung des Vertrages mit ein. Seit 2005 befragt die TK ihre Versicherten nach einem stationären Krankenhausaufenthalt zu ihrer Zufriedenheit mit der Klinik. Die Ergebnisse werden im TK-Klinikführer transparent dargestellt. Im aktuellen TKKlinikführer werden die Ergebnisse der Patientenbefragung für 624 Krankenhäuser und 1600 Fachabteilungen veröffentlicht. Hierzu wurden 263045 Versicherte befragt, von denen 157669 und damit $60 \%$ antworteten.
Die Einführung des $§ 137$ a SGB V mit der Zielstellung sektorenübergreifend abgestimmte Indikatoren und Instrumente zur Messung der Versorgungsqualität zu entwickeln, stellt einen weiteren Meilenstein für einen Vertragswettbewerb auf Grundlage von Qualitätsparametern dar.

Somit wird deutlich, dass sowohl Wettbewerb als auch Qualität in der Gesundheitsversorgung keine Gegensätze sind, sondern sich gegenseitig bedingen und befördern. So benötigt Wettbewerb in der Gesundheitsversorgung die Zieldimension Qualität und Qualität benötigt zur sachgerechten Weiterentwicklung und Steigerung einen funktionierenden Wettbewerb.

Autorenerklärung: Der Autor erklärt, dass keine relevanten finanziellen Verbindungen in Bezug auf dieses Manuskript bestehen.

\section{Literatur}

1 TK-Trendmonitor 2009 - Wünsche und Bedürfnisse der TK-Mitglieder zu Gesundheits- und Krankenversicherungsthemen; Erstellt durch das Meinungsforschungsinstitut INFO

2 Zukunft der Krankenhausversorgung: Qualität, Wettbewerb und neue Steuerungsansätze im DRG-System; Prof. Norbert Klusen und Andreas Meusch; Nomos Verlag; 2008 